\title{
Manejo da dor inguinal crônica pós-hernioplastia (inguinodinia)
}

\section{Management of chronic pain after inguinal hernioplasty}

\author{
José Guilherme Minossi, TCBC-SP1; Vinícius Vendites Minossi²; Alcino Lázaro da Silva, ECBC-MG³
}

\author{
RE S U M O
}

\begin{abstract}
A dor inguinal crônica pós-herniorrafia é uma situação preocupante, pois aproximadamente $10 \%$ dos pacientes submetidos à hernioplastia inguinal apresenta os sintomas, que com frequência limita a capacidade física. A etiopatogênese está relacionada a uma periostite do púbis (dor somática) e mais frequentemente à lesão nervosa (dor neuropática). É importante distinguir clinicamente entre os dois tipos de dor, pois o tratamento pode ser diferente. O médico deve estabelecer uma rotina diagnóstica e de tratamento, sendo que a maior parte dos pacientes necessitarão de terapêutica cirúrgica. A prevenção desta condição é de grande importância e pode levar a uma menor incidência da síndrome. Algumas medidas são fundamentais, como evitar pontos ou clipes no periósteo do púbis, usar criteriosamente as próteses e identificar os nervos da região inguinal. Esta última medida é certamente a mais importante na prevenção da dor crônica e implica em conhecimento profundo da anatomia e o uso de uma técnica aprimorada.
\end{abstract}

Descritores: Hérnia inguinal. Inguinodinia. Neuralgia crônica.

\section{INTRODUÇÃO}

A dor na região inguinal após as hernioplastias é um sintoma comum nas primeiras 24 horas, cede com o uso de analgésicos, diminui progressivamente e desaparece ao final de algumas semanas ${ }^{1}$.

Alguns pacientes queixam-se de dor constante ou intermitente com irradiação para os genitais ou porção superomedial da coxa, que os impossibilita de retornar às suas atividades habituais².

Os cirurgiões, com 20 ou mais anos de profissão, praticamente não se deparavam com esta situação. Neste período a correção dos defeitos da parede abdominal era realizada de maneira quase artesanal. As operações eram convencionais, e as técnicas de Bassini ${ }^{3}$ e Mc Vay ${ }^{4}$, as mais utilizadas. Há pouco mais de 10 anos começamos a observar queixas de dor constante após hernipolastias e em número cada vez mais crescente de publicações médicas. Existe uma certa coincidência com o uso indiscriminado das próteses para a correção destes defeitos da parede abdominal, fato que é negado por muitos autores.

Alguns cirurgiões relatam que o uso exagerado do bisturi elétrico, a falta de atenção e a pressa podem determinar lesões nervosas e um maior número de complicações nas hernioplastias.

O fato é que se a dor crônica pós-operatória da região inguinal operada não estiver relacionada a esses fatores, poderá ser decorrente da inabilidade dos cirurgi- ões em identificar e preservar os nervos da região inguinal, já que a lesão nervosa é tida como o principal fator de neuralgia crônica ${ }^{5}$.

\section{Conceito}

A dor inguinal crônica pós-herniorrafia é uma síndrome dolorosa, tardia, da região inguinal previamente operada, que se caracteriza por sensação de dor ou parestesia, e que pode se irradiar para os genitais e coxa.

\section{Classificação}

Na dor crônica pós-operatória da região inguinal deve-se distinguir três tipos': dor neuropática ou neurálgica, dor somática, "disejaculação ou deaferentação"

A dor neuropática ou neurálgica é a mais importante não só pela prevalência como pelo seu potencial incapacitante. Relaciona-se com a lesão dos nervos ilioinguinal, iliohipogástrico e genitofemoral, além do seu envolvimento no tecido cicatricial ou na formação de um neuroma.

Um segundo tipo de dor é a somática, nas inserções ligamentares do tubérculo púbico. Em repouso há uma discreta sensação álgica na inserção medial do ligamento inguinal, mas a dor é intensa com a movimentação, quando ocorre tração desse ligamento.

O terceiro tipo ("disejaculação ou deaferentação"), menos frequente $(0,04 \%)$, se caracteriza por sensação dolorosa, em queimação, durante a ejaculação.

Trabalho realizado no Departamento de Cirurgia e Ortopedia da Faculdade de Medicina de Botucatu - UNESP- SP- BR.

1. Professor Assistente Doutor do Departamento de Cirurgia e Ortopedia da Faculdade de Medicina de Botucatu - UNESP - SP-BR; 2. Acadêmico de Medicina da Universidade cidade de São Paulo - UNICID- SP-BR; 3. Professor Titular de Cirurgia do Aparelho Digestivo da Faculdade de Medicina da Universidade Federal de Minas Gerais- Belo Horizonte - MG-BR. 


\section{Prevalência}

A Prevalência da inguinodinia não está bem definida porque os métodos empregados diferem muito um dos outros.

Em nosso serviço, após análise de 493 hernioplastias inguinofemorais observamos $0,4 \%$ de dor persistente ${ }^{6}$. Atribuímos inicialmente esta baixa incidência ao fato utilizarmos seletivamente os biomateriais. Recentemente observamos um número expressivo de pacientes com dor crônica o que nos mostra a necessidade de uma nova revisão, talvez melhor sistematizada.

Wantz em 5.882 hernioplastias anteriores, sendo 5.413 pela técnica de Shouldice, teve apenas quatro casos de neuralgia crônica $(0,06 \%)^{7}$.

Ao se realizar a técnica de Shouldice, abrindo a fascia transversal, é de se esperar um número maior de lesão do ramo genital do nervo genitofemoral, com consequente incidência maior de dor crônica. Todavia ao analisar este estudo, observa-se que o autor realizava sistematicamente a secção deste nervo, motivo pelo qual os quatro casos descritos de dor foram decorrentes de neuralgia dos nervos ilioinguinal e iliohipogástrico e não do genitofemoral.

Estudo canadense multicêntrico (Cooperative Hernia Study) observou dor de média e forte intensidade em $11,9 \%$ dos pacientes submetidos às técnicas convencionais $^{8}$ ?. Outro estudo multicêntrico italiano analisou os resultados de 973 pacientes revelando que a dor crônica alcançou taxas de $9,7 \%$.

Outro aspecto controverso é a relação da dor crônica com o uso de prótese. A literatura descreve índices extremamente variáveis de 0 a $37 \%{ }^{10,11}$. Estudos multicêntricos europeus e norte-americanos indicam que o emprego de tela não se revelou um fator de aumentar a incidência de dor crônica ${ }^{12}$, embora uma revisão sistemática mais recente revele dor crônica associada ao uso de tela em $11 \%$ dos $\operatorname{casos}^{5}$ Vironen em um estudo comparativo, utilizando a técnica de Lichtenstein $(n=149)$ e PHS ( $n$ = 150), observou maior incidência com o PHS (12\%) quando comparado com Lichtenstein $(8,1 \%)^{13}$.

A inguinodinia parece ocorrer numa frequência um pouco menor nas operações por via laparoscópica, estando em 2\% a 2,5\% dos reparos ${ }^{14,15}$. Em 2.164 pacientes detectou-se dor crônica em 14,3\% nas operações abertas e em $9,8 \%$ nas laparoscópicas ${ }^{16}$.

"Embora os estudos atuais não sejam conclusivos quanto a real influência das próteses no índice de dor inguinal crônica, há indícios importantes de que o uso deste material determina uma maior incidência de inguinodinia, além de que certamente dificulta um eventual tratamento cirúrgico desta condição, uma vez que reconhecidamente aumenta a fibrose e a aderência aos tecidos" 17 .

Existe um baixo índice de diagnóstico da dor crônica ${ }^{18}$ e eventualmente o paciente é encaminhado ao psiquiatra, sem antes esgotar os algoritmos mais conhecidos para o esclarecimento da dor ${ }^{19,20}$.
Este fato revela o desconhecimento do assunto e, certamente em nosso meio deve ser ainda mais subestimada, pois os pacientes são de um modo geral humildes e com pouco acesso aos serviços especializados.

\section{Etiopatogênese}

A dor somática tem como causa, a presença de fios de sutura, clipes ou material sintético aplicado no periósteo do púbis. Um processo inflamatório (osteíte) ocasiona dor na inserção medial do ligamento inguinal e a fisiopatologia não está bem estabelecida.

A neuralgia crônica pode ser primária ou secundária. É primária quando é decorrente do trauma operatório, ou seja, secção parcial, estiramento, esmagamento, eletrocoagulação ou compressão por sutura de um dos nervos da região inguinal. O paciente permanece com dor inguinal além do período normal de recuperação pós-operatória.

A neuralgia crônica secundária aparece meses ou anos após a operação. A maioria dos casos é decorrente de fibrose perineural, que pode estar relacionada ao processo inflamatório determinado por fios de sutura ou mais frequentemente, pelo material sintético. O neuroma traumático ocorre em uma proporção menor.

\section{Quadro clínico}

A dor somática tem uma localização precisa nas inserções ligamentares do tubérculo púbico. Em repouso o paciente relata uma discreta dor na inserção medial do ligamento inguinal, mas na tração fisiológica deste ligamento, apresenta dor intensa.

A dor neuropática relaciona-se, principalmente com a distribuição dos nervos ilioinguinal, iliohipogástrico e genitofemoral. A dor, em geral, está restrita à área de inervação do nervo lesado, onde o paciente acusa dormência e certo grau de parestesia. Ela costuma ser intensa, frequentemente em queimação, podendo se acompanhar de fenômenos vegetativos e mudanças no comportamento, particularmente, depressão.

Na lesão do nervo ilioinguinal ou iliohipogástrico os sintomas usualmente envolvem a região inguinal com irradiação para a porção interna e superior da coxa, para o escroto e lábio maior.

As lesões do nervo genitofemoral também produzem sintomas na região inguinal com irradiação para a pele da genitália e parte superior e medial da coxa.

\section{Diagnóstico}

A dor somática apresenta um quadro clínico mais típico e se caracteriza por um ponto doloroso no tubérculo púbico.

A dor neurálgica se relaciona com a distribuição dos nervos na região inguinal. É intensa, em queimação e se irradia para os genitais ou face interna da coxa. A posição mais confortável é deitada com o quadril fletido. A dor é exacerbada na deambulação, quando inclinado, na 
hiperextensão do quadril ou durante as relações sexuais. Eventualmente a compressão digital de um neuroma desencadeia uma dor aguda e intensa (sinal de Tinel)..A ressonância magnética pode ser útil em alguns pacientes com suspeita de neuroma.

A eletroneuromiografia não é um método ainda bem desenvolvido, pois os nervos estabelecem anastomoses, o que dificulta a sua identificação. O exame é importante em quadros atípicos, em que a dor se irradia para uma área mais extensa, podendo indicar lesão do nervo cutâneo femoral lateral da coxa, do nervo femoral e nas radiculites por raquianestesia ${ }^{21}$

O bloqueio dos nervos abdominogenitais de um modo geral é um método importante, pois estabelece, na maioria dos casos, qual nervo está lesado.

Os nervos ilioinguinal e íliohipogástrico podem ser bloqueados dois a três centímetros medialmente à espinha ilíaca antero-superior, através da injeção de $10 \mathrm{ml}$ a $15 \mathrm{ml}$ de lidocaína a $2 \%$ sem vasoconstritor. A injeção deve ser subaponeurótica e à semelhança do procedimento utilizado para anestesia local em herniorrafias ${ }^{22}$.

Se a dor não melhorar ou houver alívio incompleto da dor, indica-se o bloqueio do nervo genitofemoral, por infiltração anestésica de $\mathrm{L}_{1}$ e $\mathrm{L}_{2}$, junto à coluna vertebral, com auxílio de um método de imagem.

Se o bloqueio do nervo genitofemoral resultar em alívio significativo da dor, o que não aconteceu após o bloqueio dos nervos ilioinguinal e iliohipogástrico, então o diagnóstico principal é o dano do nervo genitofemoral.

\section{Tratamento}

O tratamento da inguinodinia deve ser feito inicialmente com medicação antiinflamatória não hormonal ou hormonal. O uso de um corticosteróide de depósito, por via intramuscular, seguido de um antiinflamatório não esteróide pode ser uma boa opção.

A maioria dos casos necessitará de medicação específica para neuralgia crônica, como a carbamazepina ou a amitriptilina. A carbamazepina é um anticonvulsivante que tem efeito no tratamento de neuropatia periférica, como a secundária à herniorrafia ${ }^{23}$. Deve ser utilizada em doses que variam de $400 \mathrm{mg}$ a $1.200 \mathrm{mg} / \mathrm{dia}$.

A amitriptilina é um antidepressivo tricíclico, que apresenta bons resultados nos quadros álgicos crônicos, particularmente nas neuralgias periféricas. É vantajosa nos pacientes que apresentam alterações do comportamento, principalmente quadro depressivo associado. A dose usual é de $50 \mathrm{mg}$ a $150 \mathrm{mg} /$ dia. Deve-se observar seus efeitos colaterais, principalmente em idosos e cardiopatas.

A gabapentina é também um anticonvulsivante indicado na dor neuropática crônica. Os seus resultados não se mostraram superior aos demais medicamentos.

Na crioablação utiliza-se nitrogênio líquido numa temperatura de $190^{\circ} \mathrm{C}$ negativos. A crioanalgesia destrói a estrutura nervosa e cria uma degeneração waleriana, mas deixa intactos a bainha de mielina e o endoneuro ${ }^{24}$. Baseados no sucesso com o tratamento da neuralgia craniofacial e síndromes dolorosas malignas, estes autores aplicaram ablação crionalgésica na neuralgia crônica ilioinguinal e genitofemoral em 10 pacientes, com índices de sucesso em que $90 \%$ dos pacientes relataram melhora da capacidade física antes comprometida.

A radiofrequência pulsada é outra opção conversadora, sendo considerada como um tratamento não neuro-destrutivo resultando em menor potencial de formação de neuroma. Realizada em cinco pacientes com dor após hernioplastia provocou alívio da dor em quatro ${ }^{25}$.

A neuromodulação através de estimulação dos nervos periféricos com eletrodos também foi incluída neste arsenal terapêutico e pode ser lembrada como tratamento conservador em casos selecionados ${ }^{26}$.

Quando se indica o tratamento cirúrgico, passa ser importante diferenciar se a dor é somática ou neuropática. Na dor somática, em que há uma osteíte do púbis, deve-se remover material de sutura, clipes, prótese e injetar $80 \mathrm{mg}$ de metilprednisolona. Na dor neuropática pode-se indicar a neurólise ou a neurectomia.

Uma revisão sistemática realizada em 2005 mostrou ser a neurectomia o tratamento mais descrito ${ }^{27}$, registrado e com índices de sucesso de $70 \%{ }^{28}, 90 \%{ }^{29}$ e até $100 \%{ }^{30}$.

O tratamento cirúrgico da dor neuropática crônica após hernioplastia pode ser realizado em dois tempos: neurectomia ilioinguinal e íliohipogástrica, através do acesso inguinal, e neurectomia do nervo genitofemoral através de acesso no flanco. Outros têm realizado a neurectomia tripla em um só tempo, como no estudo do "Lichtenstein Hérnia Institute"12 (Los Angeles, EUA) em que 225 pacientes foram submetidos à técnica em um só tempo associando-se a implantação proximal do coto neural, evitando assim sua aderência às estruturas aponeuróticas da região inguinal, causa comum de recorrência de quadro álgico. Nesta série, o índice de cura foi semelhante à técnica em dois tempos com cura completa em $80 \%$, com 15\% de dor residual insignificante e nenhum prejuízo funcional.

Somos favoráveis a neurectomia do nervo acometido, após realizar o bloqueio anestésico. Se o bloqueio anestésico dos nervos ilioinguinal e iliohipogástrico alivia a dor, realiza-se a neurectomia desses nervos por via inguinal. Se o bloqueio desses nervos não aliviar substancialmente a dor, e o bloqueio de $L_{1}$ e $L_{2}$ resultar em considerável alívio de dor, a exploração cirúrgica do nervo genitofemoral deverá ser o primeiro caminho.

Se a dor é aliviada parcialmente com ambos os bloqueios, ou se houver dúvidas, a exploração cirúrgica deverá ser de ambos os nervos. Nesta situação é possível realizar uma inguinotomia ampliada, que fornece o acesso ao flanco e ao retroperitônio. O nervo genitofemoral pode ser identificado penetrando no músculo psoas, visivelmente como um único tronco ao longo da extremidade medial. 
Deve-se ressecar $4 \mathrm{~cm}$ a $5 \mathrm{~cm}$ do nervo genitofemoral próximo do suposto sítio de lesão, incluindo a bifurcação. Como é frequente a variação anatômica da bifurcação do nervo, ambos os ramos de nervo genitofemoral deverão ser identificados para garantir a ressecção do tronco do nervo proximalmente, ou ambos os ramos, em situação em que a bifurcação ocorre dentro da massa muscular do músculo psoas.

Logo após a entrada dos ramos do nervo genitofemoral no anel inguinal interno ou canal femoral, os ramos distais correspondentes não são precisamente identificados. A porção do nervo ilioinguinal proximal é mais frequentemente excisado durante a neurectomia genitofemoral, assim como do iliohipogástrico, que têm um trajeto próximo junto ao anel inguinal profundo.

No estudo do "Lichtenstein Hérnia Institute"12 observou-se que em $11 \%$ dos casos o achado histopatológico demonstrou neuroma traumático e em 37\% se observou fibrose perineural.

Os autores recomendam ainda sempre ligar o coto do nervo que foi ressecado para fechar a envoltura do nervo prevenindo a formação de neuroma. Se possível implantar o coto do nervo ressecado dentro das fibras do músculo oblíquo interno. Isso previne aderências desse a estruturas adjacentes e tração do mesmo aos movimentos, desencadeando dor no pós-operatório.

O paciente deve ser orientado que, após a neurectomia, a dor poderá ser substituída por áreas de hipoestesia do escroto ou grandes lábios e face interna da coxa.

A figura 1 mostra um algoritmo que pode ser seguido no manejo dos casos de inguinodinia.

\section{Prevenção}

O cirurgião deve sempre ter em mente que nada substituirá o zelo com os tecidos, o conhecimento profundo da anatomia e o uso de uma técnica aprimorada, assim atenuando o trauma cirúrgico inevitável.

Algumas medidas são de grande importância na prevenção desta síndrome:1) Evitar secção completa do músculo cremaster, pois a exposição dos elementos do cordão, para melhor tratar o saco herniário, implica em maiores riscos de lesão do nervo ilioinguinal, inclusive vascular. É aconselhável não realizar rotineiramente este procedimento, e somente faze-lo quando imprescindível para melhor exposição do saco herniário; 2) Evitar angustiar demasiadamente o anel inguinal profundo, já que os elementos do cordão devem ficar livres o suficiente para

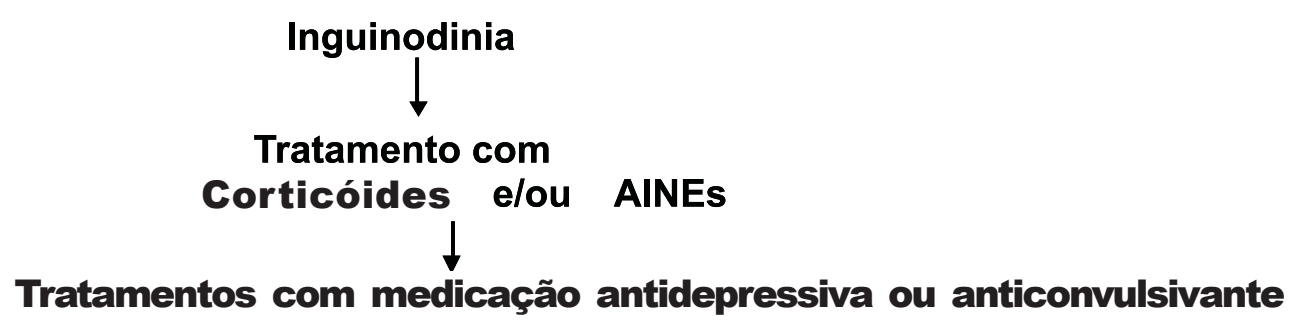

Amitriptilina (50 - $75 \mathrm{mg} / \mathrm{dia})$

Carbamazepina $\quad(800$ a $1600 \mathrm{mg} / \mathrm{dia})$
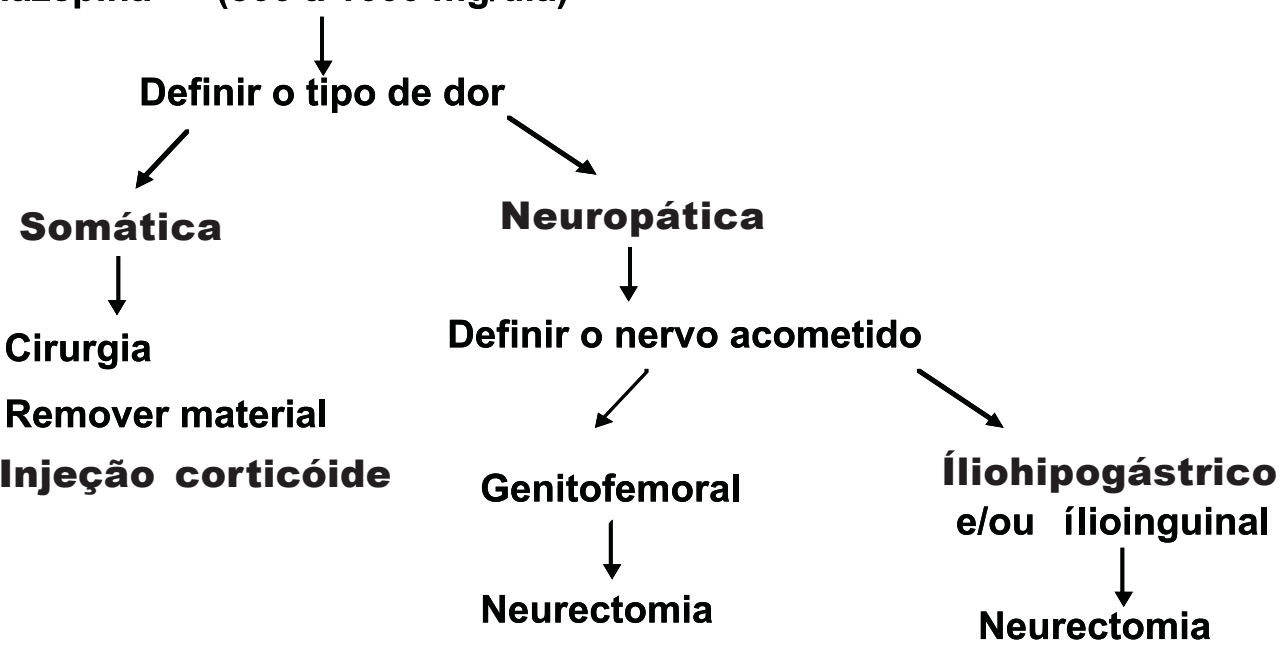

Obs 1: Na dúvida realizar neurectomia dos 3 nervos

Obs 2: Não indicar operação antes de seis meses de tratamento

Figura 1 - Algorítmo proposto para abordagem da inguinodinia. 
não sofrerem estrangulamento, tanto com a colocação de próteses como nas técnicas convencionais; 3) Identificar os nervos da região inguinal pois a falha ao identificar e proteger os nervos é reconhecida como a causa mais importante na neuralgia pós hernioplastia, . A adequada identificação de todos os nervos inguinais reduziu de forma significativa a incidência de dor crônica na hernioplastia aber$\mathrm{ta}^{31}$. ; 4) Evitar fixar pontos, clipes ou a tela na confluência do ligamento inguinal com o arco púbico (periósteo do púbis) ,tanto na hernioplastia convencional, como na laparoscópica evitando assim o aparecimento da dor somática;5) Não indicar inguinotomia exploradora pois os pacientes que têm dor inguinal e ao exame clínico não se percebe a presença de uma hérnia, deve-se procurar a causa da dor e não indicar a abordagem cirúrgica exploradora. Esses pacientes de um modo geral têm mais queixas após a internação, que antes dela; 6) Usar criteriosamente as próteses, embora o seu uso nas correções de hérnias da parede abdominal se constitua em um avanço, o seu uso indiscriminado é um abuso ${ }^{32}$, pois além de possivelmente determinar maior número de lesões nervosas, é responsável por grande número de outras complicações. Não existe lógica para a utilização de prótese nas hérnias indiretas pequenas, onde o simples tratamento do saco associado ou não a um reforço pela técnica de Marcy ou Bassini, a taxa de recidiva não ultrapassa $2 \%{ }^{32}$. Da mesma maneira não existe justificativa razoável para o uso de material sintético de rotina nas mulheres, que além de apresentarem fascia transversal resistente, a maioria das hérnias são indiretas; 7) Neurectomia preventina durante hernioplastia à Lichtenstein, mostra a preocupante a situação de dor crônica pós hernioplastia tanto que já existem autores recomendando a neurectomia de rotina durante a hernioplastia inguinal à Lichtenstein, pois estão convencidos de que mesmo com o domínio da técnica e conhecimentos da anatomia da região, a incidência de neuropatia é preocupante ${ }^{32,33}$; 8) Conduta ao observar lesão parcial de nervos: se durante a intervenção, o cirurgião observar que lesou parcialmente um dos três nervos da região inguinal, é prudente seccioná-lo e ligálo o mais alto possível, pois esta conduta pode evitar a neuralgia crônica; 9) Dominar conhecimento da anatomia regional, como único recurso para bem tratar as estruturas teciduais e preserva-las de traumas nas dissecções; 10) Não se completa o saber anatômico com colocação simples de próteses, sem antes ter a certeza sobre todos os elementos envolvidos. Um bom cirurgião, antes de tudo, deve conhecer anatomia e dominar planos de clivagem.

Em resumo, a tensão psíquica e tecidual são elementos importantes na consideração da inguinodinia. Se ela ocorre, o cirurgião não deve se esquecer de que há duas dores afetando o paciente, não lembradas com freqüência: dor fantasma e da dor da alma. Na primeira há uma associação psicossomática e na segunda a falta de cuidado, carinho e respeito pelos sentimentos e ansiedades dos pacientes que fazem com que o mais nobre no tratamento é a relação médico-paciente.

\section{Prevenção de conflitos médico legais}

Atualmente não é incomum o médico ser levado ao tribunal devido a um suposto infortúnio cometido nas operações para correção de hérnia inguinal. Frequentemente essas ações ocorrem devido a uma recidiva de hérnia, uma extrusão da tela, intervenções em lado errado ou quando se opera o paciente errado ${ }^{34}$. Recentemente o médico passou a ser acionado por paciente que apresenta dores crônicas.

O médico não será condenado se tomar alguns cuidados $^{35}$, já que o profissional tem o dever de agir com toda perícia e cautela em busca de um bom resultado, que nem sempre é certo.

Primeiramente o cirurgião que executa o procedimento, deve fazê-lo com o máximo de zelo com os tecidos, ter conhecimento profundo da anatomia e usar uma técnica aprimorada, assim atenuando o trauma cirúrgico inevitável.

O paciente deve ser informado sobre a possibilidade de dor crônica após a operação, assim como da possibilidade de recidivas e outras complicações inerentes à este tipo de intervenção. Esta informação, não deve ser feita com exageros, senão o doente pode recusar-se à intervenção, e ter as complicações próprias da doença. O médico deve orientar, ser otimista e inclusive informar que se ocorrer a dor crônica ela pode ser tratada.

Deve ser obtido sempre que possível um termo de consentimento livre e esclarecido, que embora muitos achem que este termo é uma coação, na prática tem grande valor legal, sendo aceito nos tribunais. Tem como finalidade garantir a autonomia de vontade do paciente e delimitar a responsabilidade do médico que realiza o procedimento, uma vez que desta forma cumpre com o dever de bem informar.

Na descrição cirúrgica, o cirurgião deve descrever os cuidados na identificação e preservação dos nervos e vasos da região. Deve registrar a decisão de utilizar material sintético, sua posição, e o tipo de reforço utilizado. Embora hoje seja até mais comum ocorrer uma ação judicial em situação em que não se utiliza uma tela e o paciente tem uma recidiva, já que este biomaterial tem sido descrito como indispensável por alguns autores, a nossa opinião é que não há justificativa para seu uso indiscriminado, devido aos inúmeros riscos de complicações, conforme já amplamente discutido em outro estudo ${ }^{31}$.

\section{Considerações finais}

A presença de dor crônica após a realização de uma hernioplastia inguinal é uma realidade preocupante no período atual. É esperado que sua incidência esteja em torno de $10 \%$ nas técnicas abertas e um pouco menos na operação laparoscópica.

É de grande importância algumas medidas preventivas, principalmente o zelo com os tecidos, o conhecimento profundo da anatomia e o uso de uma técnica aprimorada. 
Quando do aparecimento da dor crônica, o médico deve saber identificá-la corretamente e estabelecer uma rotina de cuidado, assistência e tratamento que, quase sempre, termina com uma internação cirúrgica.

\section{A B S T R A C T}

Chronic groin pain after herniorrhaphy is a concern, as approximately 10\% of patients undergoing inguinal hernia repair have symptoms, which often limit physical ability. The etiopathogenesis is related to periostitis pubis (somatic pain) and more often to nerve injury (neuropathic pain). It is clinically important to distinguish between these two types of pain because treatment can be different. The physician should establish a routine diagnosis and treatment, and most patients will need surgical approach. Prevention of this condition is of great importance and can lead to a lower incidence of the syndrome. Some measures are key, such as how to avoid application of stitches or clips to the pubis periosteum, using the prosthesis carefully and identifying the nerves in the groin. This last measure is certainly the most important in the prevention of chronic pain and involves thorough knowledge of anatomy and the use of refined technique.

Key words: Inguinal hernia. Chronic inguinal pain. Somatic pain. Neuropathic pain.

\section{REFERENCIAS}

1. Speranzini $M B$, Deutsch $C R$, editores. Tratamento cirúrgico das hérnias das regiões inguinal e crural. 1a ed. São Paulo: Atheneu; 2001. Complicações da cirurgia corretiva das hérnias; p.101-14.

2. Rodrigues BDS, Lázaro-da-Silva A. Hérnias. 2ª ed. São Paulo: Rocca; 2006. Inguinodinia; p.594-7.

3. Bassini E. Nuovo metodo per la cura radicale dell'Ernia inguinale. Atti Cong Ass Med Ital 1887;2:179-82.

4. McVay CB. Inguinal and femoral hernioplasty: anatomic repair Arch Surg 1948;57(4):524-30.

5. Nienhuijis S, Staal E, Strobbe L, Rosman C, Groenewoud H, Bleichrodt R. Chronic pain after mesh repair of inguinal hernia: a systematic review. Am J Surg 2007;194(3):394-400.

6. Minossi JG, Kemp R, Picanco HC, spadella CT. Avaliação pós-operatória tardia de pacientes submetidos à herniorrafia inguinocrura por via anterior, utilizando a técnica convencional ou a colocação de prótese sintética. ABCD arq. bras.cir.dig 2004;17(4):163-9.

7. Wantz GE. Complications of inguinal hernial repair. Surg Clin North Am 1984;64(2):287-98.

8. Cunnigham J, Temple WJ, Mitchell P, Nixon JA, Preshaw RM, Hagen NA. Cooperative Hernia Study. Pain in the postrepair patient. Ann Surg 1996;224(5):598-602

9. Alfieri S, Rotondi F, Di Giorgio A, Fumagalli U, Salzano A, Di Miceli D, Ridolfini MP, Sgagari A, Doglietto G, Groin Pain Trial Group. Influence of preservation versus division of ilioinguinal, iliohypogastric, and genital nerves during open mesh herniorrhaphy: prospective multicentric study of chronic pain. Ann Surg 2006;243(4): 553-8

10. Lichstenstein IL, Shulman AG, Amid PK, Montllor MM. Cause and prevention of postherniorrhaphy neuralgia: a proposed protocol for treatment. Am J Surg 1988;155(6):786-90.

11. Laparoscopic versus open repair of groin hernia: a randomized comparison. The MRC Laparoscopic Groin Hernia Trial Group. Lancet 1999:354(9174):185-90.

12. Amid PK. Causes, prevention, and surgical treatment of postherniorrhaphy neuropathic inguinodynia: triple neurectomy with proximal end implantation. Hernia 2004;8(4):341-9.

13. Vironen J, Nieminen J, Eklund A, Paavolainen P. Randomized clinical trial of Lichtenstein patch or Prolene Hernia System for inguinal hernia repair. Br J Surg 2006;93(1):33-9.

14. Callesen T, Bech K, Nielsen R, Andersen J, Hesselfeldt P, Roikjaer $\mathrm{O}$, Kehlet $\mathrm{H}$. Pain after groin hernia repair. $\mathrm{Br} J$ Surg 1998;85(10):1412-14.

15. Hay JM, Boudet MJ, Fingerhut A, Poucher J, Hennet H, Habib E, Veyrières $M$, Flamant $Y$. Shouldice inguinal hérnia repair in the male adult: the gold standard? A multicenter controlled trial in 1578 patients. Ann Surg 1995;222(6):719-27

16. Neumayer L, Giobbie-Hurder A, Jonasson O, Fitzgibbone R Jr, Dunlop D, Gibbs J, Reda D, Henderson W, Veterans Affair Cooperative Studies Program 456 Investigators. Open mesh versus laparoscopic mesh repair of inguinal hernia. N Engl J Med 2004;350(18):1819-27.

17. Bendavid R. Complications of groin hernia surgery. Surg Clin North Am 1998;78(6):1089-103.

18. McGarrity TJ, Peters DJ, Thompson C, McGarrity SJ. Outcome of patients with chronic abdominal pain referred to chronic pain clinic. Am J Gastroenterol 2000:95(7):1812-6.

19. Thompson C, Goodman R, Rowe WA. Abdominal wall syndrome: A costly diagnosis of exclusion. Gastroenterology 2001;120 (5):A637.

20. Ferraz ED. Neuropatia por aprisionamento entidade não esquecida na era laparoscópica. Rev bras videocir 2007;5(3):144-57.

21. Ferrari FG, Castiglia YMM, Ganem EM, Resende LAL. Lesão do nervo femoral após cirurgia para correção de hérnia inguinal: relato de caso. Rev bras anestesiol 2000;50(4):297-8.

22. Minossi JG, Pedro FAJ, Vendites S. Herniorrafia inguinal com anestesia local. Arq gastroenterol 1992:29(1):18-22.

23. Rizzo MA. Successful treatment of painful traumatic mononeuropathy with carbamazepine: insights into a possible molecular pain mechanism. J Neurol Sci 1997;152(1):103-6.

24. Fanelli RD, DiSiena MR, Lui FY, Gersin KS. Cryoanalgesic ablation for the treatment of chronic postherniorrhaphy neuropathic pain. Surg Endosc 2003;17(2):196-200.

25. Rozen D, Ahn J. Pulsed radiofrequency for the treatment of ilioinguinal neuralgia after inguinal herniorrhaphy. Mt Sinai J Med 2006;73(4):716-8.

26. Paicius RM, Bernstein CA, Lempert-Cohen C. Peripheral nerve field stimulation in chronic abdominal pain. Pain Physician 2006;9(3):261-6

27. Aasvang $E$, Kehlet $H$. Surgical management of chronic pain after inguinal hernia repair. Br J Surg 2005;92(7):795-801.

28. Stulz P, Pfeiffer KM. Peripheral nerve injuries resulting from common surgical procedures in the lower portion of the abdomen. Arch Surg 1982;117(3):324-7.

29. Kim DH, Murovic JA, Tiel RL, Kline DG. Surgical management of 33 ilioinguinal and iliohypogastric neuralgias at Lousiana State University Health Sciences Center. Neurosurgery 2005;56(5):101320; discussion 1013-20.

30. Murovic JA, Kim DH, Tiel RL, kline DG. Surgical Managementof 10 genitofemoral neuralgias at the Lousiana Stante University Health Sciences Center. Neurosurgery 2005;56(2):298-303; discussion 298-303. 
31. Wijsmuller AR, van Veen RN, Bosch JL, Lange JF, Kleinrensink GJ, Jeekel J, Lange JF. Nerve management during open hernia repair. $\mathrm{Br}$ J Surg 2007;94(1):17-22.

32. Minossi JG, Silva AL, Spadella CT. O uso da prótese na correção das hérnias da parede abdominal é um avanço, mas o seu uso indiscrimado, um abuso. Rev Col Bras Cir [online]. 2008;35(6):41623.

33. Dittrick GW, Ridl K, Khun JA, McCarty TM. Routine ilioinguinal nerve excision in inguinal hernia repairs. Am J Surg 2004;188(6):73640.

34. Minossi JG, Silva AL. Aspectos médico-legais da cirurgia para hérnia inguinal. Rev Col Bras Cir 2005;32(4):214-7.

35. Minossi JG. Prevenção de conflitos médico-legais no exercício da medicina. Rev Col Bras Cir 2009;36(1):90-5
Recebido em 15/10/2009

Aceito para publicação em 10/12/2009

Conflito de interesse: nenhum

Fonte de financiamento: nenhuma

\section{Como citar este artigo:}

Minossi JG, Minossi VV, Silva AL. Manejo da dor inguinal crônica póshernioplastia (inguinodinia). Rev Col Bras Cir. [periódico na Internet] 2011; 38(1). Disponível em URL: http://www.scielo.br/rcbc

\section{Endereço para correspondência:}

José Guilherme Minossi

E-mail: jmossi@uol.com.br / jminossi@fmb.unesp.br 